\title{
Brightness constancy in a Ganzfeld environment
}

\author{
GERARD SCHOUTEN and FRANS J. J. BLOMMAERT \\ Institute for Perception Research (IPO), Eindhoven, The Netherlands
}

\begin{abstract}
In this paper, experiments are described in which brightness constancy was studied in a Ganzfeld environment. Luminance variation by means of neutral density filters was applied to stimuli consisting of a Ganzfeld with superimposed disks. To this end, a special-purpose apparatus was constructed. Sequential dichoptical brightness matches with a reference stimulus were carried out for the disks as well as the homogeneous surround. The results of these measurements indicate that (1) besides a clear tendency toward brightness constancy, small but systematic effects of the average luminance level are present and (2) the brightness of the Ganzfeld is hardly affected by the presence of the disks. Finally, it is shown that the experimental results can be modeled adequately in terms of a concept that involves an accumulation of contrast information.
\end{abstract}

The human visual system has the capacity to respond to an enormous range of light intensities. Hecht, Shlaer, and Pirenne (1942) showed that people can reliably detect a few photons. Bartley (1951) reported that the luminance ratio of snow on a mountaintop illuminated by the sun to the Hecht et al. absolute threshold is ten billion to one. It can therefore be stated safely that the human visual system is able to handle a 10-decade luminance range. On the other hand, there is converging evidence that the corresponding sensorial range (i.e., the brightness range) is considerably smaller (e.g., Barlow \& Verrillo, 1976; Marks, 1974; Whittle, 1992). As a consequence, the visual system must accomplish a substantial compression. Brightness constancy can be regarded as the appearance of a sensible compression mechanism (Forsyth, 1990; Schouten, 1993; Schouten \& Blommaert, 1991; Walraven, Enroth-Cugell, Hood, MacLeod, \& Schnapf, 1990).

Usage of the term brightness constancy needs some explanation. Although, according to the literal meaning, brightness constancy should be reserved to express invariance with respect to changes of the illumination level, we will relax that strict condition to insensitivity for illumination. One of the first psychophysical experiments demonstrating the brightness-constancy phenomenon was published almost a century ago by two of Hering's students, Hess and Pretori (1894). ${ }^{1}$ Among other important, earlier contributions concerning brightness constancy are the studies of MacLeod (1932), Wallach (1948), Heinemann (1955), Jameson and Hurvich (1961), and Bartleson and Breneman (1967). Contemporary research related to the constancy principle is interwoven with the lightnessbrightness controversy concerning the argument that lightness and brightness are two distinct attributes of achromatic color perception (McCann, 1989). Brightness is used to refer to perceived luminance. Lightness is interpreted as

Correspondence should be addressed to G. Schouten, Philips CFT, Building SAQ-2, P.O. Box 218, $5600 \mathrm{MD}$ Eindhoven, The Netherlands (e-mail: schouten_g@cft.philips.nl). apparent reflectance, ranging from black through grays to white. Arend and Goldstein (1987), Jacobsen and Gilchrist (1988), and Arend and Spehar (1993a) showed that if the illumination level is varied, lightness constancy is nearly perfect, and that this does not hold to the same extent for brightness. The term brightness constancy has increasingly been abandoned in the recent literature - in fact, we severely hesitate to reintroduce it. The most important reason to maintain the traditional term brightness constancy, however, is to emphasize that, in conformity with the aforementioned studies, brightness is to a large extent determined by luminance ratios. We certainly do not want to deny that there are photometrical components involved in brightness perception.

In the present investigation, brightness constancy is verified for images that consisted of a Ganzfeld on which disks were superimposed. A Ganzfeld environment is used because this configuration provides a very useful stimulus for tackling the anchoring problem systematically (see Gilchrist \& Bonato, 1995; Gilchrist, Cattaliotti, Bonato, \& Li, 1992). Perfect brightness constancy would imply that luminance ratios are transferred to the brightness scale. As such, this mapping is ambiguous. If, additionally, the brightness of at least one point in the image can be identified, the constancy principle can be used to disambiguate other parts of the image. For brightness perception, there has been little explicit treatment of the anchoring problem. Assumptions known as the gray-world hypothesis (Hurlburt, 1986) or the assignment of white to the highest scene luminance (Land \& McCann, 1971) can be seen as specific rules for lightness perception.

\section{Brightness Constancy: Principle and Practice}

The concept of perfect brightness constancy is visualized in Figure 1. Two luminance distributions that are equal except for a constant factor are mapped onto identical brightness representations. In other words, perfect brightness constancy reflects invariance for changes of the average luminance level. It is clear that in order to describe a perfect constancy mapping completely, an anchoring rule is 


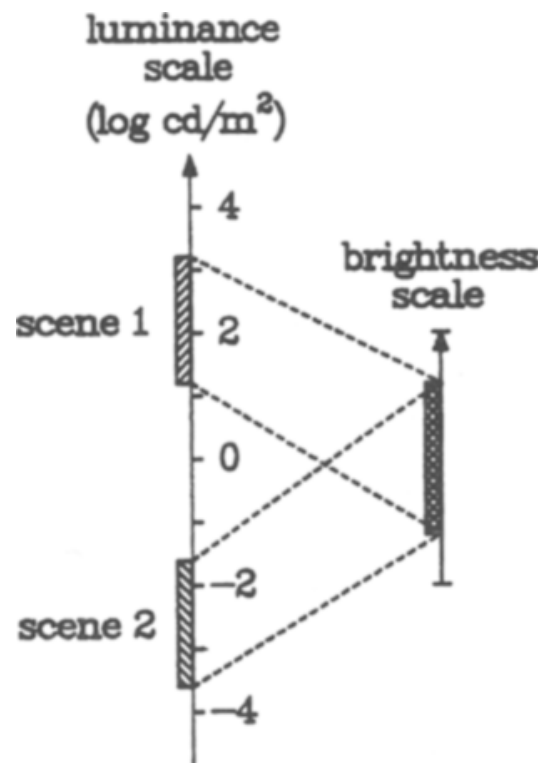

Figure 1. The principle of brightness constancy. Scene 2 is an attenuated version of Scene 1 . The shift on the logarithmic luminance axis mimics the effect of putting on sunglasses.

necessary. Somehow, at least one luminance value (derived from the image) must be tied to a specific location on the brightness scale.

A critical look at the aforementioned publications on brightness constancy reveals that all of these measurements display deviations, to a greater or lesser extent, from perfect brightness constancy; that is, there is a dependence on the average luminance level. At this point, a few comments can be made. In many psychophysical experiments, only a relatively small part of the visual field was manipulated (e.g., a $10^{\circ} \times 10^{\circ}$ square corresponding to a monitor display). Specification of the environment is often given in rather vague terms, such as "the experimental room was otherwise dimly lit," or "the stimuli were presented in the dark." It is obvious that this typical laboratory situation differs from most natural circumstances. Moreover, in the case of brightness-constancy experiments, luminance variation by means of a multiplier (usually neutral density filters) has been explicitly applied within a limited part of the visual field. Without further preface, it is therefore not clear whether the luminance statistics of the whole visual field shifted in the desirable way (see Figure 1). To avoid these problems, an extended, controllable surround is necessary. For this reason, a specialpurpose optical apparatus has been designed.

\section{Brightness Versus Lightness}

The CIE vocabulary (CIE, 1970) $)^{2}$ employs the following definitions of brightness and lightness: BrightnessAttribute of visual sensation according to which an area appears to emit more or less light. This attribute is the psychosensorial correlate, or nearly so, of the photometric quantity luminance. Lightness-Attribute of visual sensation in accordance with which a body seems to transmit or reflect diffusely a greater or smaller fraction of the incident light. This attribute is the psychosensorial correlate, or nearly so, of the photometric quantity luminance factor. ${ }^{3}$ Although the definitions in themselves are clear, in the past, the actual usage of both terms has been sloppy. The modern opinion is that brightness and lightness are two simultaneously available attributes of achromatic color perception (see, e.g., Arend \& Spehar, 1993a; McCann, 1989)-in other words, that independent estimations of luminances as well as luminance factors (reflectances) can be made. Because this was not realized in the classical studies cited in the introduction, the results of those studies are often ambiguous. It is not clear whether observers judged brightness, lightness, or some mixture of both.

A direct and elegant demonstration of a fundamental difference between brightness and lightness is presented by Schirillo, Reeves, and Arend (1990). They showed that lightness matches are influenced by perceived depth, whereas brightness matches are not. In other recent work of Arend and Goldstein $(1987,1990)$ and Arend and Spehar (1993a, 1993b), the above distinction between brightness and lightness was also explicitly made. Their data indicate that specific brightness and lightness tasks produce significantly different results. Since we are primarily interested in brightness perception, our observers were asked to judge "brightness or perceived luminance, i.e., the apparent light-density." This instruction must be looked upon as opposed to a typical lightness task in which observers are asked to judge stimuli "as if they were cut from a similar piece of paper."

\section{METHOD}

\section{Apparatus}

With a special-purpose apparatus based on the integrating sphere principle, which is also frequently employed in photometric measuring instruments (Billmeyer, 1989; Le Grand, 1968), an adjustable, homogeneous environment was created. A schematic drawing of this optical apparatus is presented in Figure 2. We attached to the front of the sphere a cap that fits around the orbit of the observer's left eye. The sphere is illuminated by means of a xenon lamp (XBO, 150W/1). After passing through the appropriate optics, light enters by a small hole covered with a piece of tracing paper that transmits diffusely. The central part of the observer's field of view is filled in with the display of a monitor (Barco CTVM 3/51). By using a diffusing screen (Cinemoid No. 29 heavy frost) to obscure the visible line structure of the display, and by adjusting the monitor's luminance and color coordinates, ${ }^{4}$ a uniform field of view (Ganzfeld) can be presented to the viewer's left eye

We took two precautions in order to reduce the visibility of remaining conspicuous inhomogeneities. First, the visible transition between the monitor and sphere was defocused by putting a frostedglass hemisphere with a central hole close to the viewer's left eye. This hole corresponds to a $17.7^{\circ}$ circular field of view. In this construction, the newly created transition, between the monitor and the hemisphere, is not visible, since it is out of the normal eye's accommodation range and therefore heavily blurred. Second, local spatial inhomogeneities perceived as vague light spots (e.g., at the position where the beam of the xenon light source enters the sphere) were covered by glued masking elements ${ }^{5}$ with about the same reflecting properties as the inner-sphere surface. Calibration measurements 


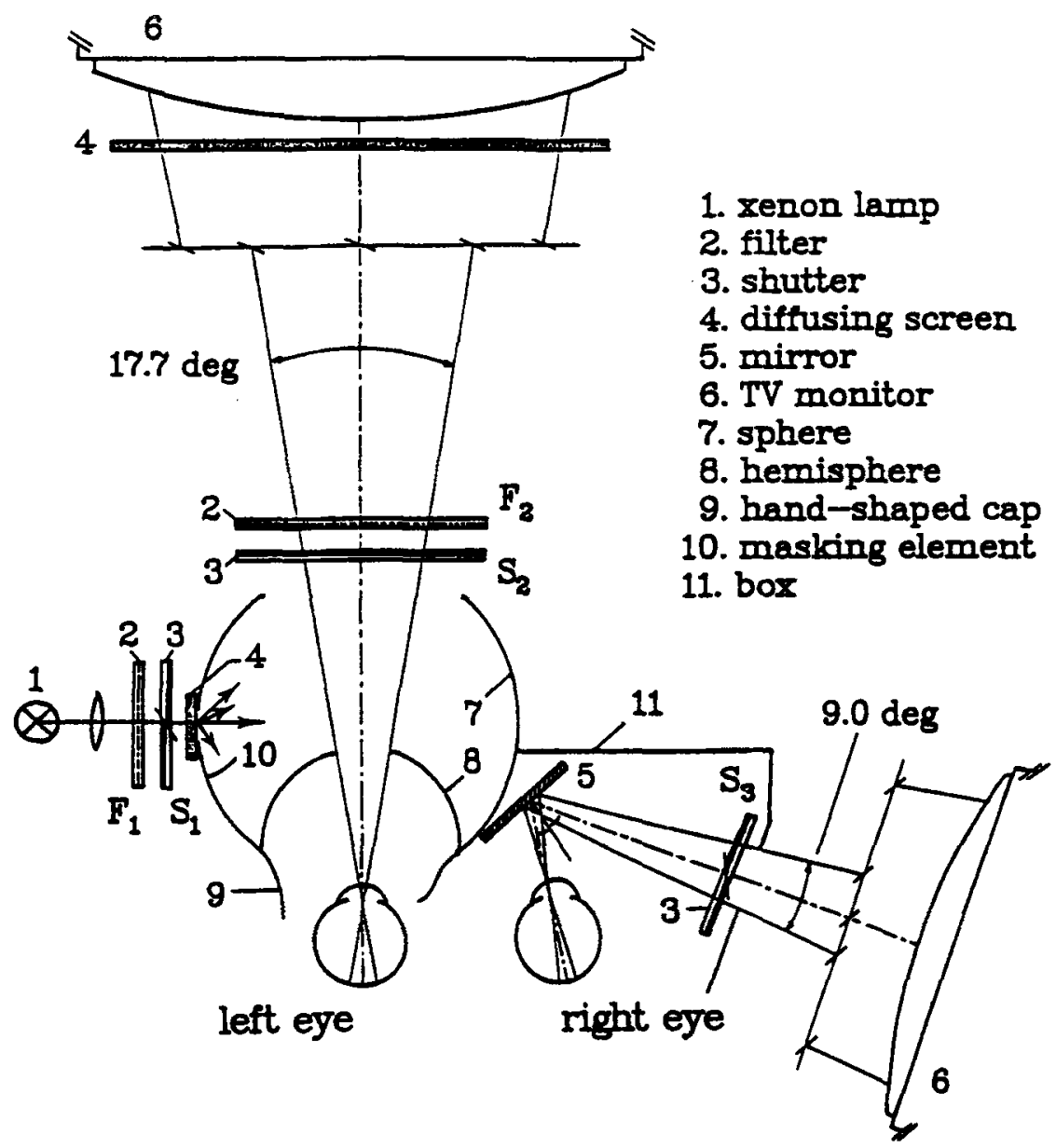

Figure 2. Special-purpose optical apparatus.

with a luminance meter (PR-1980A Pritchard) on this subjectively homogeneous field of view showed a luminance decline from the center to the periphery varying between $5 \%$ and $9 \%$ per $30^{\circ}$.

Neutral density filters, $F_{1}$ and $F_{2}$, can be used to attenuate, respectively, the large surround and the $17.7^{\circ}$ circular field. We positioned coupled shutters, $S_{1}$ and $S_{2}$, at the indicated positions to switch off (or on) the total field of view of the viewer's left eye. The shutters are controlled by external electronic equipment. The advantage of this apparatus in comparison with the traditional ping-pong-ball construction (e.g., Barlow \& Verrillo, 1976; Corwin \& Green, 1978; Hochberg, Triebel, \& Seaman, 1951) is that arbitrary luminance distributions can be placed in the visual field, independently of an approximately constant, adjustable surround.

The image of another monitor (Barco TVM 3/37) is projected via a mirror to the viewer's right eye. Both monitors are connected to a Gould-deAnza image-processing device. A cardboard box, wrapped in black velvet, was built around the mirror to screen indirect lighting. The mirror's position can be changed to compensate for differences in interocular distances between observers. Exposure to the right eye is controlled by a shutter $\left(\mathrm{S}_{3}\right)$ with a $9^{\circ}$ circular aperture. The viewer's head is supported by a chinrest.

\section{General Procedure}

The Ganzfeld image presented to the viewer's left eye is called the test stimulus. The right-eye image will be designated the reference stimulus. The spatial configuration of the reference stimulus is given in Figure 3. It consisted of a $1.2^{\circ}$ disk with a variable luminance $L_{\text {ref }}$ on a $9^{\circ}$ background field with a fixed luminance $L_{0, \mathrm{ref}}\left( \pm 3 \mathrm{~cd} / \mathrm{m}^{2}\right)$ in an otherwise dark surround. This enables both incremental and decremental settings of the small reference disk to be made with respect to the adjacent background field.

In the experiments, the observer's task was to adjust the luminance $L_{\text {ref }}$ of this reference disk until it was perceived as being equal to the brightness of a previously indicated field of the test stimulus. It should be emphasized that in order to obtain unambiguous match data, we adopted the Arend and Spehar (1993a, 1993b) brightnessmatch task rather literally. Observers were explicitly instructed "to make the brightness, i.e., the apparent light-density, of the reference disk and the relevant test field equal, disregarding other areas in the visual field as much as possible. Potential color and texture differences between the test field and the reference disk have to be ignored." Effects of small color shifts on brightness matches are considered separately in Appendix A.

The left-eye test stimulus and the right-eye reference stimulus were presented sequentially with dark periods in between. The time sequence of the brightness matches is sketched in Figure 4. Most of the time, shutters $S_{1}$ and $S_{2}$ were open and $S_{3}$ was closed, so that the observer viewed the test stimulus. When a start button was pressed, $S_{1}$ and $S_{2}$ closed for $3 \mathrm{sec}$. After $1 \mathrm{sec}$ of darkness, $S_{3}$ opened for $1 \mathrm{sec}$, during which the observer saw the reference stimulus. When $S_{1}$ and $S_{2}$ opened again (i.e., when the test stimulus reappeared), the observer had to report whether the reference disk was darker or brighter than the specific test field. In doing so, he tried to abstract brightness values from the indicated regions and compare them on an internal scale. After the experimenter had adjusted the luminance $L_{\text {ref }}$ of the reference disk, the trial was repeated by operating the start 


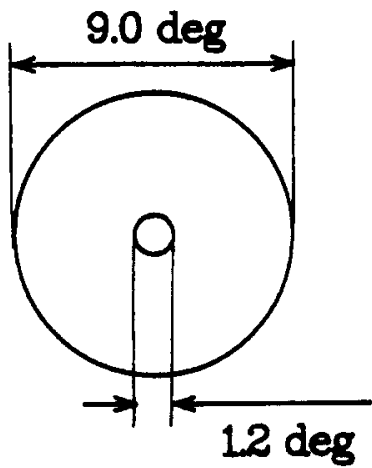

Figure 3. Spatial configuration of the reference stimulus.

button again until a match was obtained. More precisely, a staircase procedure with randomized starting points was used to converge to the ultimate $L_{\text {ref }}$ value. The minimum step in $\log L_{\text {ref }}$ (a measure of the match accuracy) is about 0.015 . On average, the start button was operated once every $10 \mathrm{sec}$. About eight trials were needed to arrive at a match. The effect of this specific time sequence was evaluated in a general control experiment (see Appendix B).

Prior to each experimental session, the observer first adapted to the dark for about $15 \mathrm{~min}$, and then adapted his left eye to the test stimulus for another $5 \mathrm{~min}$. Pupillary aperture was not controlled and fixation was not guided.

\section{Observers}

Three observers participated in the experiments. All had uncorrected vision. Two of them were well trained but had mild red-green defects. As diagnosed by the H-R-R test (Hardy, Rand, \& Rittler, 1957), Observer F.B. was deuteranomalous, while Observer G.S. proved to be protanomalous. Observer M.N. was unexperienced. At the time of the experiments, two observers, G.S. and M.N., were 27 years old, while the third, F.B., was 42 . All three observers were well aware of the difference between brightness and lightness.

\section{Test stimuli and procedure}

As usual for brightness-constancy experiments, luminances were varied with a multiplier. In general, the following routine was applied. First, several points of a test stimulus with luminance distribution $L(x, y)$ were matched with an adjustable reference. Then the luminance distribution $L(x, y)$ was replaced by another luminance distribution $L^{\prime}(x, y)$ that can be written as

$$
L^{\prime}(x, y)=\kappa \cdot L(x, y),
$$

where $\kappa$ is a scalar, and brightness matches involving the same points in the scene are again performed. This is repeated for a number of $\kappa$ values. For our experimental setup, a Ganzfeld was created (see Apparatus section). Subsequently, two disks with different luminances were positioned on the central circular region. Changes in $\kappa$ were accomplished by means of neutral density filters, $F_{1}$ and $F_{2}$, having the same light transmission. Owing to the design of the optical apparatus shown in Figure 2, the above pointwise attenuation formula applies explicitly to the entire field of view.

The test stimuli used in this experiment consisted of two $5.3^{\circ}$ disks in an otherwise uniform surround, as illustrated in Figure 5. The angular distance between the central points of these disks was $10.6^{\circ}$. To describe the test stimuli, it is sufficient to consider the situation for one value of $\boldsymbol{k}$. In that case, only three luminance values are present. By mutual exchange of these luminance values over the scene elements, three patterns, $\mathrm{P}_{1}, \mathrm{P}_{2}$, and $\mathrm{P}_{3}$, were constructed.

At the end of an experimental session, in which only a single pattern was used, one brightness match (i.e., one value of $L_{\text {ref }}$ ) was obtained for every scene element and $\kappa$ value. For each pattern, there were three scene elements and six different $\kappa$ values spanning a 3decade luminance range. This yielded a total of 18 matches per session. To shorten the duration of the experimental sessions as much as possible, we started with the most attenuated situation and proceeded with larger and larger $\kappa$ values. This is because light adaptation progresses quickly in comparison with dark adaptation. A sequence effect might be introduced by starting with the most attenuated situation and proceeding with larger and larger $\kappa$ values (i.e., by working from "dark" towards "light" stimuli). However, a few control matches for decreasing $\kappa$ values with time (see Figure 7) demonstrate that effects of sequence are negligible. Within each session, the experimenter determined the order in which the various elements of the test stimulus were matched with the reference disk. A session lasted approximately $45 \mathrm{~min}$. On one day, three sessions, one for each pattern, were run in an arbitrary order. Between sessions, the observer was given a considerable amount of time to recuperate. Eventually, four brightness matches were collected for every $\kappa$ value and scene element of each pattern, leading to 12 sessions for each observer.

\section{RESULTS}

The results of these measurements are shown in Figure 6. The symbols in the plots represent geometrical means of four $L_{\text {ref }}$ settings. Standard deviations of these means exceeding the symbol size are marked by vertical bars. Pattern $P_{1}$ is denoted by triangles, $P_{2}$ by circles, and $\mathrm{P}_{3}$ by squares. In all cases, open symbols are for either Disk 1 or Disk 2 (see Figure 5). Filled symbols always refer to brightness matches of the Ganzfeld. Matches of scene elements belonging to the same luminance distribution are connected; a dashed line is used for $P_{1}$, a solid line for $\mathrm{P}_{2}$, and a dotted line for $\mathrm{P}_{3}$. The constant background luminance $L_{0 \text {, ref }}$ of the reference stimulus is denoted by a star on the ordinate.

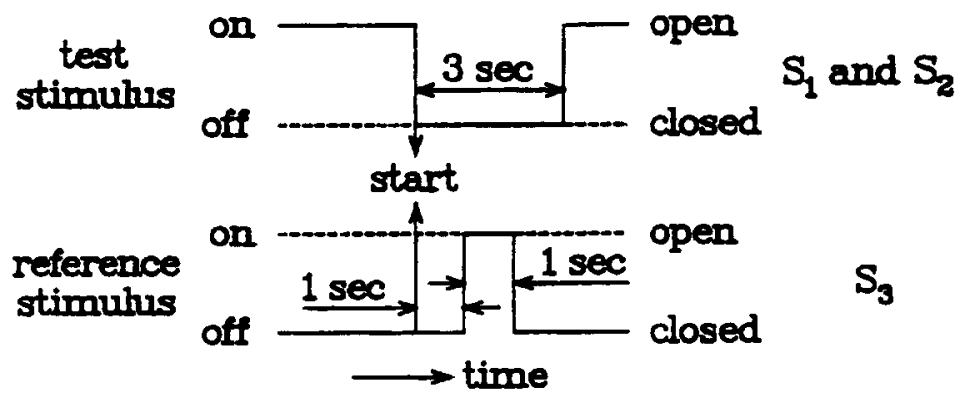

Figure 4. Time sequence of the brightness matches. 

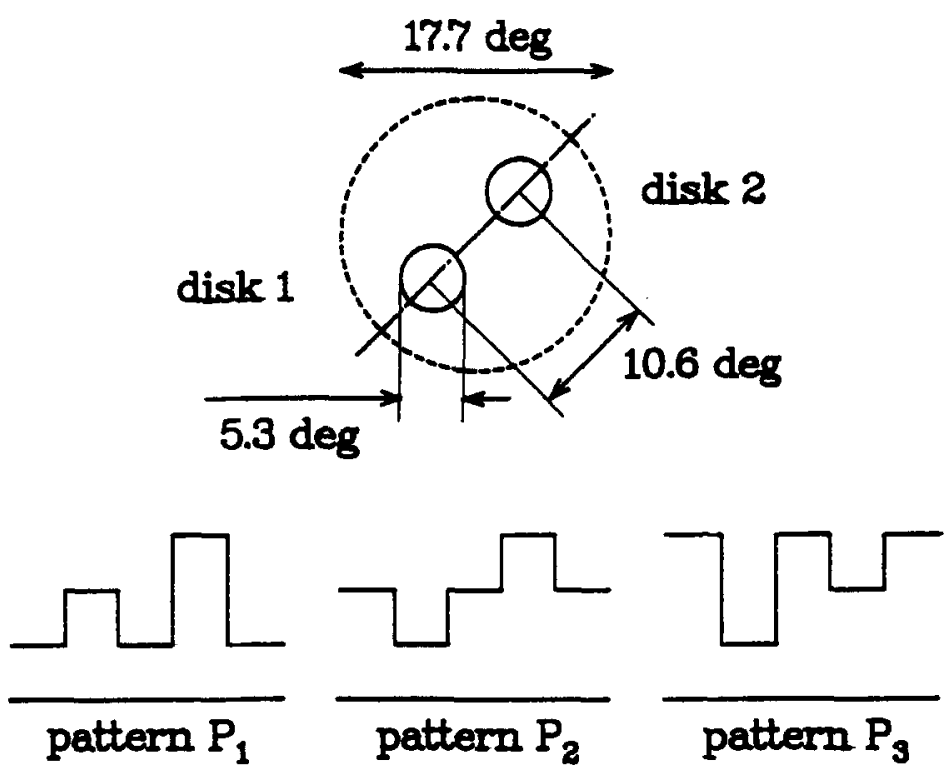

Figure 5. Top: Spatial configuration of the test stimuli. Bottom: Cross-section along the diagonal for each of the three luminance patterns $P_{1}, P_{2}$, and $P_{3}$. The dashed circle marks the invisible transition between monitor and hemisphere (see Figure 2).

The data display several trends. Since brightness increases monotonically with the luminance of the reference disk, the $\log L_{\text {ref }}$ axis is in topological correspondence with the brightness scale. Therefore, the vertical axis in Figure 6 can be interpreted as a (perhaps warped) brightness yardstick. The following can now be concluded:

1. At first sight, brightness constancy nearly holds. Whereas the luminances of the patterns $P_{1}, P_{2}$, and $P_{3}$ vary over three decades, $\log L_{\text {ref }}$ changes, on average, merely by a few tenths. To illustrate this clearly, the data of Observer F.B. for Pattern $\mathrm{P}_{2}$ are isolated and plotted in Figure 7. Compared with the variation of the Ganzfeld brightness, the dark disk, marked by circles with a horizontal line, displays no systematic brightness change and the bright disk, marked by circles with a vertical line, causes a relatively large displacement of about 0.5 log-units on the ordinate. Consequently, brightness differences become gradually smaller as $\kappa$ decreases. Furthermore, the very fact that we can speak about dark and bright disks, despite substantial luminance variations according to Equation 1, already reflects brightness-constancy behavior. In this connection, note that, as indicated by the arrows in Figure 7, the luminance of the bright disk at the lowest $\kappa$ value is almost one-hundredth of the luminance of the dark disk at the highest $\kappa$ value.

2. The brightness of the Ganzfeld is hardly affected by the presence of the disks. In other words, to a firstorder approximation, the Ganzfeld brightness serves as an anchorage. The plots in Figure 6 demonstrate that the brightness matches of the uniform surround, no matter which pattern is regarded, can be depicted onto a single straight line. The slope of this line differs between observers.

\section{ANALYSIS}

\section{Theory}

In many theoretical frameworks, it is assumed that brightness (or lightness) can be assembled from contrasts. The following are some examples. The retinex algorithm (Land, 1986; Land \& McCann, 1971) accumulates logarithmic luminance ratios along some path in the visual field. The more physiologically oriented approach of Grossberg and Todorovic (1988), which is in essence similar, prefers the terminology "boundary segmentation by contrast-sensitive units followed by a filling-in process." In the object-oriented brightness model (Blommaert \& Martens, 1990; Blommaert, Schouten, \& Martens, 1989), contrast information is represented within a multiplescale structure. Brightness is computed by a summation over the scales. It is important to remark that all of these models intrinsically include perfect brightness constancy.

In order to incorporate the occurring deviations from perfect brightness constancy into this broad class of models, consider the following expression in which the brightness, $B$, of a scene element is written as a weighted sum of contrasts:

$$
B=\sum_{i} \alpha_{i} C_{i}
$$

Note that within this general scheme, $B=0$ corresponds to the absence of contrast information. In principle, dif- 

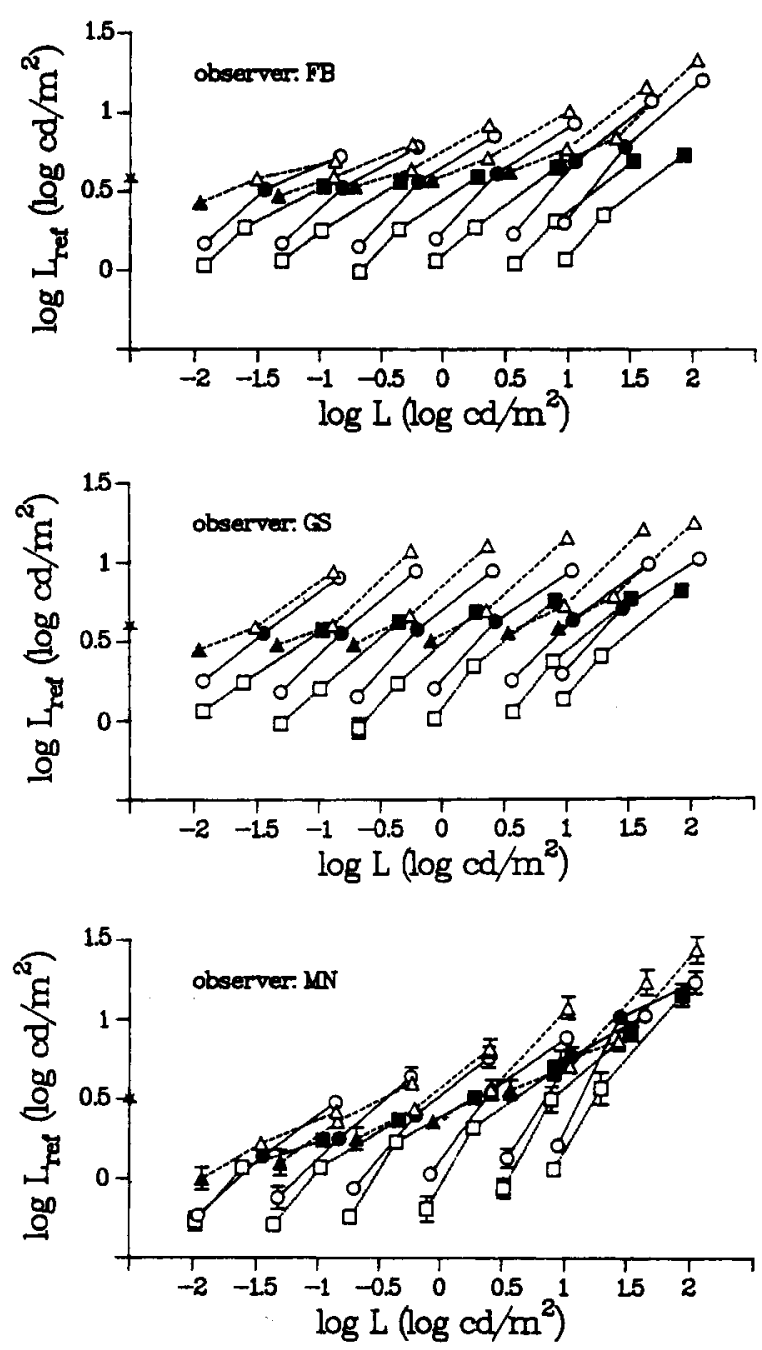

Figure 6. Results of the brightness-constancy measurements for Observers F.B., G.S., and M.N. Luminances of the scene elements in the patterns $P_{1}, P_{2}$, and $P_{3}$ are plotted along the horizontal axis. The vertical axis represents the luminance of the reference disk. The background luminance of the reference stimulus is denoted by a star. The symbols in the plots represent geometrical means of four matches. Standard deviations of these means are indicated by vertical bars. Pattern $P_{1}$ is marked by triangles, $P_{2}$ by circles, and $P_{3}$ by squares. Open symbols are for either Disk 1 or Disk 2. Filled symbols refer to brightness matches of the Ganzfeld. Matches of scene elements belonging to the same luminance distribution are connected; a dashed line is used for Pattern $P_{1}$, a solid line for $P_{2}$, and a dotted line for $P_{3}$.

ferent contrast measures (e.g., Moulden, Kingdom, \& Gatley, 1990) can be taken for $C_{i}$ (e.g., Weber contrast, Michelson contrast, retinex contrast, etc.). Contrast definitions have the common feature that they all reflect a measure for the spatial variation in luminance with respect to some average level. Therefore, contrast is invariant for luminance changes that obey Equation 1 (i.e., it does not depend on illumination level). To give concrete form to the above concept, we adopt in this paper the retinex-contrast mea- sure, which is defined as the logarithm of a luminance ratio (Land 1986). To be precise, the retinex contrast $C_{i}$ of an object with luminance $L_{i}$ on a background with luminance $L_{0}$ equals

$$
C_{i}=\log \frac{L_{i}}{L_{0}} .
$$

On the other hand, the weights $\alpha_{i}$ may vary with illumination. Obviously, within this scheme, deviations from perfect brightness constancy are attributed to variations in $\alpha_{i}$ as a function of luminance. The weights $\alpha_{i}$ can be interpreted as efficiency factors.

\section{Application to the Brightness-Constancy Experiment}

Before applying this concept to our brightness-constancy measurements, one additional postulate is necessary. Within the context of Equation 2, the brightness of a Ganzfeld is ill defined. Since a Ganzfeld is contourless, there is in principle no contrast information present. However, the results of the experiment show not only that the Ganzfeld brightness $B_{\mathrm{G}}$ is independent of the presence of the disks and gradually increases as a function of its luminance $L_{\mathrm{G}}$, but also that Ganzfelder behave in conformity with the smaller disks. Therefore, we propose that the brightness $B_{\mathrm{G}}$ originates from a fixed contrast value $C_{\mathrm{G}}$ derived from the Ganzfeld luminance and a hypothetical internal reference level,

$$
B_{\mathrm{G}}=\alpha_{\mathrm{G}} C_{\mathrm{G}},
$$

where $\alpha_{\mathrm{G}}$ is the efficiency factor of the Ganzfeld. Now, the brightness $B_{\text {test }}$ of any element of the test stimulus (the Ganzfeld or one of the disks) can be written as

$$
B_{\text {test }}=\alpha_{\mathrm{G}} C_{\mathrm{G}}+\alpha_{\mathrm{d}} C_{\mathrm{d}}
$$

Here, $C_{\mathrm{d}}=\log \left(L_{\mathrm{d}} / L_{\mathrm{G}}\right)$ refers to the contrast between the considered scene element (disk), having a luminance $L_{\mathrm{d}}$, and the uniform surround. $\alpha_{\mathrm{d}}$ denotes the corresponding

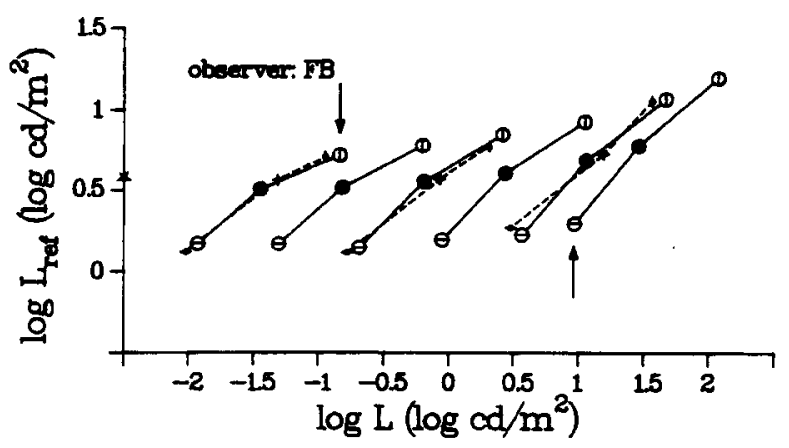

Figure 7. Brightness-constancy measurements of Observer F.B. for Pattern $P_{2}$, copied from Figure 6 . Here, the dark disk is denoted by a circle filled with a horizontal line, the bright disk by a circle filled with a vertical line. For clarity, these lines are omitted in the graphs of Figure 6. Some control matches for decreasing $\kappa$ values with time are also plotted. These are denoted by small symbols connected with dashed lines. 
weight. If $C_{\mathrm{d}}=0\left(L_{\mathrm{d}}=L_{\mathrm{G}}\right)$ is substituted, the Ganzfeld brightness obviously emerges. Similarly, application of Equation 2 to the reference stimulus leads to the following expression for the brightness $B_{\text {ref }}$ of the reference disk:

$$
B_{\text {ref }}=B_{0, \text { ref }}+\alpha_{\text {ref }} C_{\text {ref }} .
$$

In this formula, $C_{\text {ref }}$ represents the contrast between the reference disk and its $9^{\circ}$ background field. Likewise, $C_{\text {ref }}$ $=\log \left(L_{\text {ref }} / L_{0, \text { ref }}\right)$, where $L_{\text {ref }}$ and $L_{0, \text { ref }}$ denote the luminances of the reference disk and the constant background field, respectively. Again, $\alpha_{\text {ref }}$ refers to the weighting factor. The brightness $B_{0 \text {, ref }}$ of this background field equals a weighted sum of the remaining, constant contrasts related to the reference stimulus.

As a first-order approximation, we assume that the factors $\alpha$ of the test stimulus depend only on the Ganzfeld luminance $L_{\mathrm{G}}$, that is,

$$
\alpha_{\mathrm{G}}=\alpha_{\mathrm{d}}=\alpha_{\mathrm{test}}\left(L_{\mathrm{G}}\right) .
$$

Encouraged by the tendencies in the experimental data (see Figure 6), we propose for $\alpha_{\text {test }}\left(L_{\mathrm{G}}\right)$ a linear function of $\log L_{\mathrm{G}}$ :

$$
\alpha_{\text {test }}\left(L_{\mathrm{G}}\right)= \begin{cases}0 & \text { for } L_{\mathrm{G}}<L_{\mathrm{th}}, \\ \hat{\alpha} \log \left(L_{\mathrm{G}} / L_{\mathrm{th}}\right) & \text { for } L_{\mathrm{G}} \geq L_{\mathrm{th}} .\end{cases}
$$

The luminance $L_{\mathrm{th}}$ can be interpreted as a threshold level. When the luminance is smaller than $L_{\mathrm{th}}$, the visual system cannot obtain any contrast information at all. Furthermore, for the reference stimulus, $\alpha_{\text {ref }}$ and $B_{0 \text {, ref }}$ are regarded as constants.

Brightness matching between the reference disk and a scene element of the test stimulus implies that $B_{\text {ref }}=B_{\text {test }}$. Substitution of Equations 5 and 6, together with the function given in Equation 8, yields the important expression

$$
C_{\text {ref }}=\frac{\hat{\alpha}}{\alpha_{\text {ref }}}\left(\log L_{\mathrm{G}}-\log L_{\mathrm{th}}\right)\left(C_{\mathrm{G}}+C_{\mathrm{d}}\right)-\frac{B_{0, \mathrm{ref}}}{\alpha_{\mathrm{ref}}}
$$

In this expression, $\hat{\alpha} / \alpha_{\mathrm{ref}}, \log L_{\mathrm{th}}, C_{\mathrm{G}}$, and $B_{0 \text {, ref }} / \alpha_{\mathrm{ref}}$ are the four unknown parameters; $\log L_{\mathrm{G}}$ and $C_{\mathrm{d}}$ can be considered as the input variables, determined by or derived from physical measurements at the test stimulus; and $C_{\text {ref }}$ represents the output variable (i.e., the predicted contrast between the reference disk and its background field).

The model summarized by Equation 9 is fitted to the data points of the brightness-constancy measurements. Note that the ordinate of Figure 6 can be converted into a vertical $C_{\text {ref }}$ axis, simply by offsetting in such a way that the star, representing $\log L_{0, \text { ref }}$, coincides with $C_{\text {ref }}=0$ (see Figure 8). Parameter values were estimated separately for each observer. This was done by minimizing the sum of squared differences between predicted $C_{\text {ref }}$ values and experimental $C_{\text {ref }}$ values. The estimated parameters and their standard deviations are listed in Table 1 . The result of the fit of the model to the brightness-constancy measurements is shown in Figure 8. In these graphs, data points are plotted as a function of $\log L_{\mathrm{G}}$ (for a description of the symbols, refer to the caption of Figure 6). From Equation 9, it is clear that, for a fixed value of $C_{\mathrm{d}}$ and for $L_{\mathrm{G}}>$ $L_{\text {th }}$, a straight line with slope $\hat{\alpha} / \alpha_{\text {ref }}\left(C_{\mathrm{G}}+C_{\mathrm{d}}\right)$ results. Furthermore, it can easily be verified that a set of such lines, each labeled with a different $C_{\mathrm{d}}$ value, possesses one common point of intersection-namely, $\left(\log L_{\mathrm{th}},-B_{0, \mathrm{ref}} / \alpha_{\mathrm{ref}}\right)$. The displayed fanlike structure through the data points is generated with the estimated parameters. The dashed lines refer to the predictions for pattern $P_{1}$, while the solid lines refer to those for $P_{2}$, and the dotted lines to those for $P_{3}$.

Although, as can be seen in Figure 8, a reasonable overall fit is obtained for all three observers, some minor discrepancies are present. In particular, predicted $C_{\text {ref }}$ values for the dark disk of Pattern $\mathrm{P}_{2}$ tend to lie above the data points. This is elucidated once more in Figure 9. Here, slopes $a$ of regression lines of data points in Figure 8 that belong to a certain scene element of either $P_{1}, P_{2}$, or $P_{3}$ and run through the point of intersection $\left(\log L_{\mathrm{th}}\right.$, $\left.-B_{0 \text {, ref }} / \alpha_{\text {ref }}\right)$ are compared with the performance of the model-that is, $\hat{\alpha} / \alpha_{\text {ref }}\left(C_{\mathrm{G}}+C_{\mathrm{d}}\right)$.

It is worth mentioning that despite an extrapolation of more than 3 decades, the estimated values of $\log L_{\mathrm{th}}$ correspond to published measurements of the absolute threshold ( $\pm 3 \times 10^{-6} \mathrm{~cd} / \mathrm{m}^{2}$; see, e.g., Bartley, 1951). Further, it is striking that the parameter sets of Observers M.N. and, especially, F.B. display smaller variances in comparison with the parameter set of Observer G.S. The reasons for the poorly conditioned parameter set of $\mathrm{Ob}$ server G.S. are twofold. First, unlike those for Observers F.B. and M.N., the straight lines through the data points of Observer G.S. have a clear tendency to run parallel. This means that the point of intersection, which directly concerns the parameters $\log L_{\mathrm{th}}$ and $B_{0 \text {, ref }} / \alpha_{\text {ref }}$, is determined with a low accuracy. Second, in the case of Observer G.S., the brightness matches of the Ganzfeld do not fit neatly onto a single line, as dictated by the model. Moreover, a small contextual effect can be seen; at a fixed value of $\log L_{\mathrm{G}}$, the experimental $C_{\text {ref }}$ value of the uniform surround of Pattern $P_{1}$ is smaller than the experimental $C_{\text {ref }}$ value of the uniform surround of Pattern $P_{2}$, which in turn is smaller than the experimental $C_{\text {ref }}$ value of the uniform surround of Pattern $\mathrm{P}_{3}$. The fact that the standard devia-

Table 1

Estimated Model Parameters and Corresponding Standard Deviations for Each Observer

\begin{tabular}{lcccccccc}
\hline Observer & $\hat{\alpha} / \alpha_{\text {ref }}$ & $S D$ & $\log L_{\text {th }}$ & $S D$ & $C_{\mathrm{G}}$ & $S D$ & $B_{0, \text { ref }} / \alpha_{\text {ref }}$ & $S D$ \\
\hline F.B. & 0.102 & \pm 0.013 & -5.07 & \pm 0.66 & 0.90 & \pm 0.14 & 0.49 & \pm 0.08 \\
G.S. & 0.026 & \pm 0.016 & -21.2 & \pm 12.9 & 2.59 & \pm 1.61 & 1.46 & \pm 0.90 \\
M.N. & 0.097 & \pm 0.022 & -6.29 & \pm 1.49 & 2.54 & \pm 0.60 & 1.60 & \pm 0.39 \\
\hline
\end{tabular}



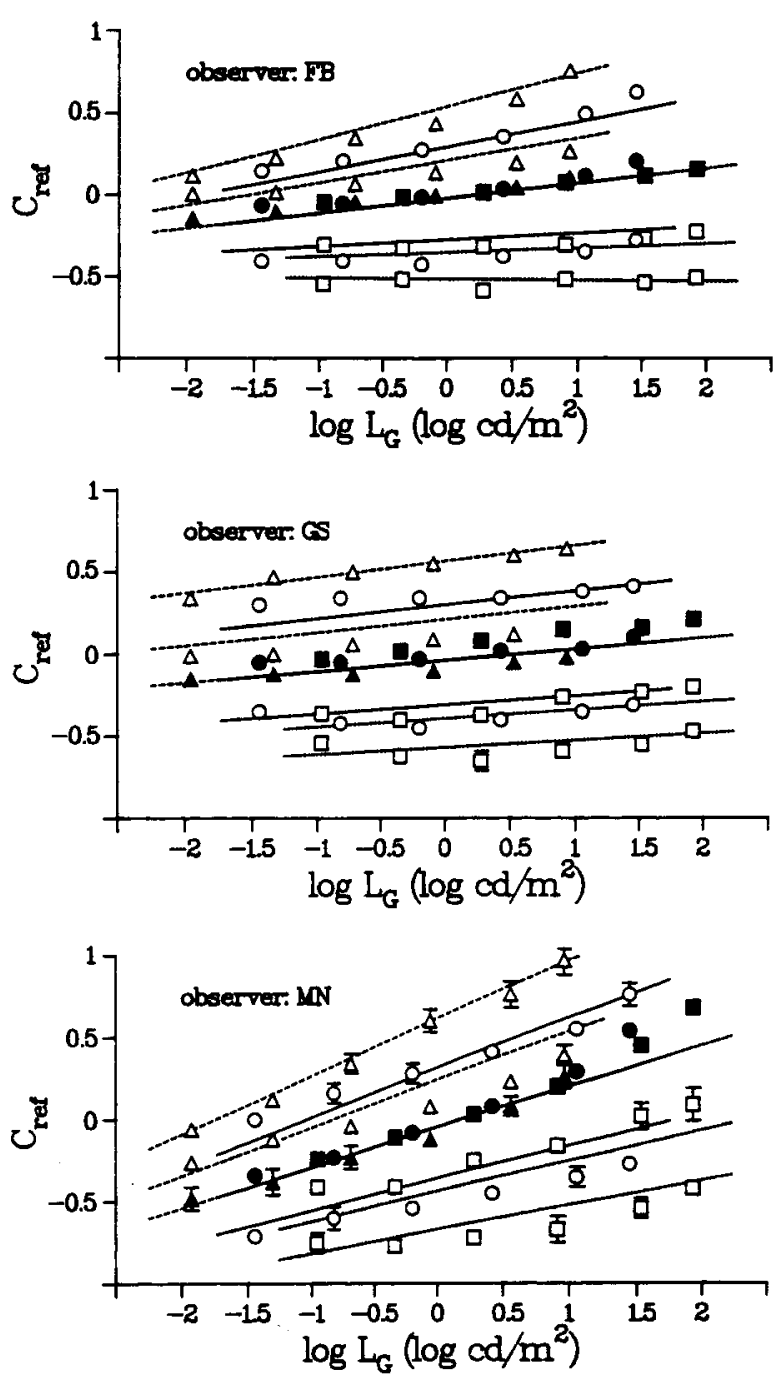

Figure 8. Experimental results as a function of $\log L_{\mathrm{G}}$ together with curves generated by the model, for Observers F.B., G.S., and M.N. Dashed curves are for Pattern $P_{1}$, solid curves for $P_{2}$, and dotted curves for $P_{3}$. A description of the symbols is given in the caption of Figure 6.

tions of the parameters for Observer M.N. are somewhat larger than those for Observer F.B. is due to noise on the measurements, which, in the case of Observer M.N., is considerably greater.

\section{DISCUSSION}

First of all, it can be stated that, like classical data (e.g., Bartleson \& Breneman, 1967; Jameson \& Hurvich, 1961), our experiment concerning images with a Ganzfeld surround demonstrates brightness-constancy behavior. Although luminances of the test stimuli were varied over about 3 decades, $\log L_{\text {ref }}$ changes span merely a few tenths. It is evident that this points to a powerful compression in the luminance-brightness mapping. On the other hand, there is a small but systematic effect of luminance. Bright- ness differences within a scene are magnified when the factor $\kappa$ of Equation 1 increases. Experimental support for this is provided by Arend (1993), Peli, Yang, Goldstein, and Reeves (1991), and Whittle and Challands (1969), who showed that perceived contrast diminishes at low luminance levels. It is also in agreement with the fact that the Weber fraction of a stimulus is not exactly constant but gradually increases if the luminance is reduced (Blackwell, 1946). Clearly, the displayed brightness-constancy behavior is not the same for all three observers. The most conspicuous difference is that for Observer M.N., the luminance dependence is somewhat more pronounced, at the cost of the constancy principle. Comparable individual differences are reported by, for instance, Arend (1993) and Arend and Spehar (1993a, 1993b).

Second, it is shown that the brightness of the Ganzfeld, which is, to a first-order approximation, independent of the superimposed disks, slowly increases with its luminance. Barlow and Verrillo (1976) and Bolanowski (1987), using the method of magnitude estimation, determined brightness variations of a pure Ganzfeld over an 8-decade luminance range. Their results indicate that subjective estimates can be described by power functions of luminance with exponents of around 0.3. De Ridder (1992), Krueger (1989), and Wagenaar (1982), for example, pointed out that this might be overestimated owing to nonlinear number handling of human observers. A closer look at the Ganzfeld matches also reveals a second-order contextual effect (see also Birnbaum, 1982), present especially in the data of Observer G.S.

The findings can be modeled satisfactorily with a simple theoretical framework that basically involves an accu-
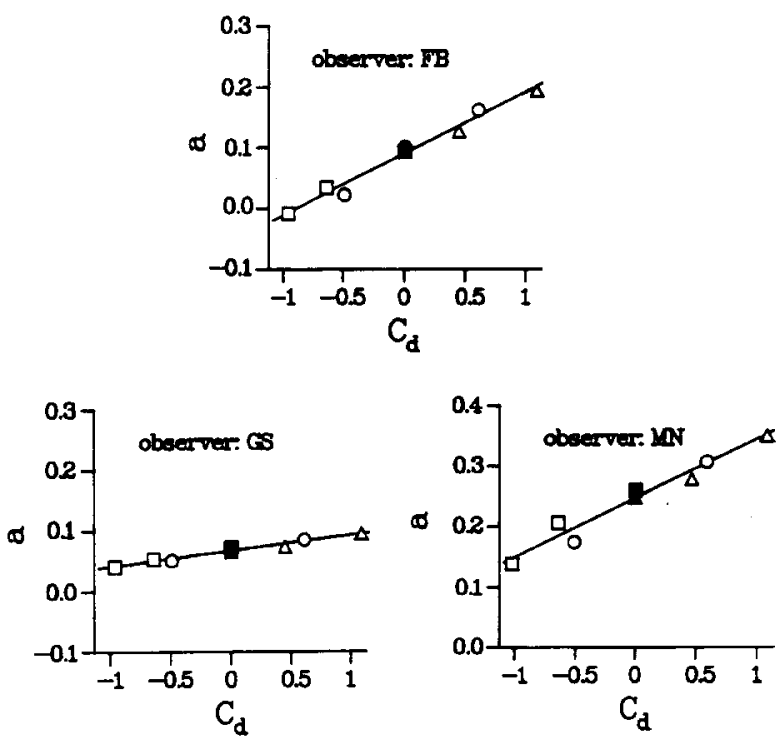

Figure 9. Slopes of regression lines through the estimated intersection point as a function of the contrast $C_{d}$, for Observers F.B., G.S., and M.N. In accordance with Figures 6 and 8, triangles are for Pattern $P_{1}$, circles for $P_{2}$, and squares for $P_{3}$. Filled symbols refer to the Ganzfeld. Standard deviations of the slopes are smaller than the symbol size. The solid line reflects the behavior of the model. 
mulation of contrast information. Within this concept, built-in weights are used to cope with the luminance dependence. It is argued that the characteristic Ganzfeld behavior can be incorporated easily by including an additional contrast with an internal reference level. Many authors (e.g., Blommaert \& Martens, 1990; Blommaert et al., 1989; Forsyth, 1990; Walraven et al., 1990, to cite some recent references) have already emphasized the logic and functional role of constancy mechanisms: They link perception to object properties. One can also speculate on functional arguments concerning the luminancedependent component. Our measurements indicate that the human visual system has, on account of brightness information, two clues for estimating the illumination. The first is the average brightness of a scene. For the test stimuli used in the experiments, this average level approximately equals the Ganzfeld brightness. The second cue, a more indirect one, may be expressed as the vividity of a scene, and involves a measure for the occurring brightness differences. It can indeed be verified from introspection that we can see whether, for instance, the lighting in a room is comfortable. Likewise, we know if it is broad daylight or dimming toward twilight. Probably, it is by means of these weak photometrical characteristics that brightness representations, resulting from different images, can be compared with one another. Alternatively, the luminance dependence might also point to what Marr (1982) calls "the principle of graceful degradation," providing a gradual decline of contrast vision toward nil in the absence of light.

\section{REFERENCES}

AREND, L. E. (1993). Mesopic lightness, brightness, and brightness contrast. Perception \& Psychophysics, 54, 469-476.

AREND, L. E., \& GoldSTEIN, R. (1987). Simultaneous constancy, lightness, and brightness. Journal of the Optical Society of America, A, 4, 2281-2285.

AREND, L. E., \& Goldstein, R. (1990). Lightness and brightness over spatial illumination gradients. Journal of the Optical Society of America, $A, 7,1929-1936$

AREND, L. E., \& Spehar, B. (1993a). Lightness, brightness, and brightness contrast: 1. Illuminance variation. Perception \& Psychophysics, 54, 446-456.

AREND, L. E., \& SPEHAR, B. (1993b). Lightness, brightness, and brightness contrast: 2. Reflectance variation. Perception \& Psychophysics, 54, 457-468.

Barlow, R. B., \& VerRillo, R. T. (1976). Brightness sensation in a Ganzfeld. Vision Research, 16, 1291-1297.

Bartleson, C. J., \& Breneman, E. J. (1967). Brightness perception in complex fields. Journal of the Optical Society of America, 57, 953957.

BartLEY, S. H. (1951). The psychophysiology of vision. In S. S. Stevens (Ed.), Handbook of experimental psychology (p. 945). New York: Wiley.

BILLMEYER, F. (1989). The measurement of luminous flux (CIE Publication No. 84). Vienna: CIE Central Bureau.

BIRNBAUM, M. H. (1982). Controversies in psychological measurement. In B. Wegener (Ed.), Social attitudes and psychophysical measurement (pp. 40 -482). Hillsdale, NJ: Erlbaum.

BLACKWELl, H. R. (1946). Contrast thresholds of the human eye. Journal of the Optical Society of America, 36, 624-643.

Blommaert, F. J. J., \& Martens, J. B. (1990). An object-oriented model for brightness perception. Spatial Vision, 5, 15-41.

Blommaert, F. J. J., Schouten, G., \& Martens, J. B. (1989). An object-oriented brightness model. IPO Annual Progress Report, 24, $61-71$

Bolanowski, S. J. (1987). Contourless stimuli produce binocular brightness summation. Vision Research, 27, 1943-1951.

CIE (1970). International lighting vocabulary (CIE Publication No. 17). Paris: Bureau Central de la CIE.

Corwin, T. R., \& GreEN, M. A. (1978). The Broca-Sulzer effect in a Ganzfeld. Vision Research, 18, 1675-1678.

DE RIDDER, H. (1992). A ratio scale for brightness perception derived from difference and ratio judgments (Report No. 869). Eindhoven, The Netherlands: Institute for Perception Research.

FLOCK, H. R., \& TENNEY, J. H. (1970). Quantitative investigation of the lawfulness of simultaneous brightness contrast. Perceptual \& Motor Skills, 31, 947-969.

Forsyth, D. A. (1990). Colour constancy. In A. Blake \& T. Troscianko (Eds.), Al and the eye (pp. 201-229). Chichester, U.K.: Wiley.

Gilchrist, A., \& Bonato, F. (1995). Anchoring of lightness values in center/surround displays. Manuscript submitted for publication.

Gilchrist, A., Cattaliotti, J., Bonato, F., \& Li, X. (1992). What is the anchoring rule for lightness perception? Perception, 21(Suppl. 2), 15.

Grossberg, S., \& Todorović, D. (1988). Neural dynamics of 1-D and 2-D brightness perception: A unified model of classical and recent phenomena. Perception \& Psychophysics, 43, 241-277.

HaRdy, L. H., RAND, G., \& RitTleR, M. C. (1957). H-R-R pseudoisochromatic plates, for detecting, classifying, and estimating the degree of defective color vision. New York: American Optical Company.

Hecht, S., Shlaer, S., \& Pirenne, M. H. (1942). Energy, quanta and vision. Journal of General Physiology, 25, 819-840.

HeinemanN, E. G. (1955). Simultaneous brightness induction as a function of inducing- and test-field luminances. Journal of Experimental Psychology, 50, 89-96.

Hess, C., \& Pretori, H. (1894). Messende Untersuchungen über die Gesetzmässigkeit des simultanen Helligkeits-contrastes [Quantitative investigation of the lawfulness of simultaneous brightness contrast]. Albrecht von Graefe's Archiv für Ophthalmologie, 40, 1-24.

Hochberg, J. E., Triebel, W., \& Seaman, G. (1951). Color adaptation under conditions of homogeneous visual stimulation (Ganzfeld). Journal of Experimental Psychology, 41, 153-159.

HurLbert, A. (1986). Formal connections between lightness algorithms. Journal of the Optical Society of America A, 3, 1684-1693.

JACOBSEN, A., \& GILCHRIST, A. (1988). The ratio principle holds over a million-to-one range of illumination. Perception \& Psychophysics, 43, 1-6.

Jameson, D., \& Hurvich, L. M. (1961, January). Complexities of perceived brightness. Science, 133, 174-179.

KRUEGER, L. E. (1989). Reconciling Fechner and Stevens: Toward a unified psychophysical law. Behavioral \& Brain Sciences, 12. 251-320

LAND, E. H. (1986). Recent advances in retinex theory. Vision Research, 26, 7-21.

LAND, E. H., \& MCCANN, J. J. (1971). Lightness and the retinex theory. Journal of the Optical Society of America, 61, 1-11.

LE GRAND, Y. (1968). Light, colour and vision. London: Chapman \& Hall

MACLEOD, R. B. (1932). An experimental investigation of brightness constancy. Archives of Psychology, No. 135.

MarKs, L. E. (1974). Sensory processes-The new psychophysics. New York: Academic Press.

Marr, D. (1982), Vision. San Francisco: W. H. Freeman

MCCANN, J. J. (1989). The role of nonlinear operations in modeling human lightness and color sensation. Proceedings of the SPIE: Human Vision, Visual Processing, and Digital Display, 1077, 355-363.

Moulden, B., Kingdom, F., \& Gatley, L. F. (1990). The standard deviation of luminance as a metric for contrast in random-dot images. Perception, 19, 79-101.

Peli, E., Yang, J., Goldstein, R., \& Reeves, A. (1991). Effect of luminance on suprathreshold contrast perception. Journal of the Optical Society of America, $A, \mathbf{8}, 1352-1359$.

Schirillo, J., Reeves, A., \& Arend, L. (1990). Perceived lightness, but not brightness, of achromatic surfaces depends on perceived depth information. Perception \& Psychophvsics, 48, 82-90.

SCHOUTEN, G. (1993). Luminance-brightness mapping: The missing decades. Unpublished doctoral thesis, Eindhoven University of Technology, Eindhoven, The Netherlands. 
Schouten, G., \& Blommaert, F. J. J. (1991). Mapping luminance into brightness: Identification of compression mechanisms. IPO Annual Progress Report, 26, 61-69.

WAGENAAR, W. A. (1982). Misperception of exponential growth and the psychological magnitude of numbers. In B. Wegener (Ed.), Social attitudes and psychophysical measurement (pp. 283-300). Hillsdale, NJ: Erlbaum.

WALLACH, H. (1948). Brightness constancy and the nature of achromatic colors. Journal of Experimental Psychology, 38, 310-324.

Walrayen, J., Enroth-Cugell, C., Hood, D. C., MacLeod, D. I. A., \& SCHNAPF, J, L. (1990). The control of visual sensitivity: Receptoral and postreceptoral processes. In L. Spillman \& J. S. Werner (Eds.), The neurophysiological foundations of visual perception ( $\mathrm{pp}$. 53-100). London: Academic Press.

WHITrLE, P. (1992). Brightness, discriminability and the "crispening effect." Vision Research, 32, 1493-1507.

WhitTle, P., \& Challands, P. C. D. (1969). The effect of background luminance on the brightness of flashes. Vision Research, 9, 10951110.

\section{NOTES}

1. An English translation of this article, with some comment added, is given by Flock and Tenney (1970).

2. In the 1987 edition, a slightly different description of lightness is proposed. However, we prefer the 1970 definitions.

3. Ratio of the luminance of the body to that of a perfect reflecting or transmitting diffuser identically illuminated.

4. The optimal setting is close to the color coordinates of the Dilluminant (see Figure $1 \mathrm{~A}$ ).

5. Parts of a ping-pong ball are suitable.

\section{APPENDIX A}

\section{Control Experiment 1: Effects of Color}

The reasons for checking on interference of color and brightness are twofold. First, all three observers complained about the difficulty of matching a scene element of the test stimulus with the reference disk when color differences were present. Particu-

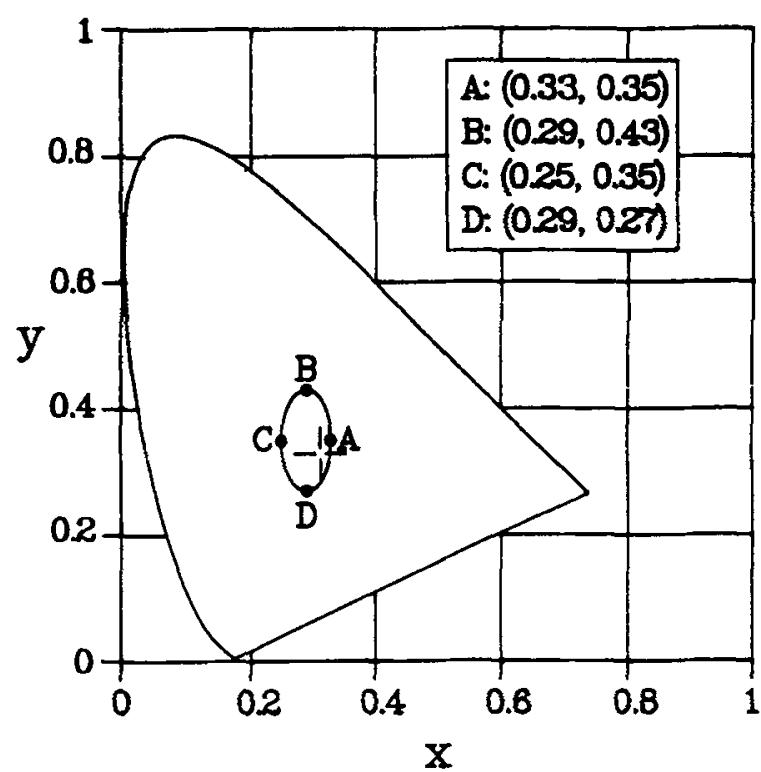

Figure A1. CIE chromaticity diagram. The color points of the test stimuli are marked as $A, B, C$, and $D$. The color point of the reference disk coincides with $A$. The $D$-illuminant is located in the center of the pinnule.
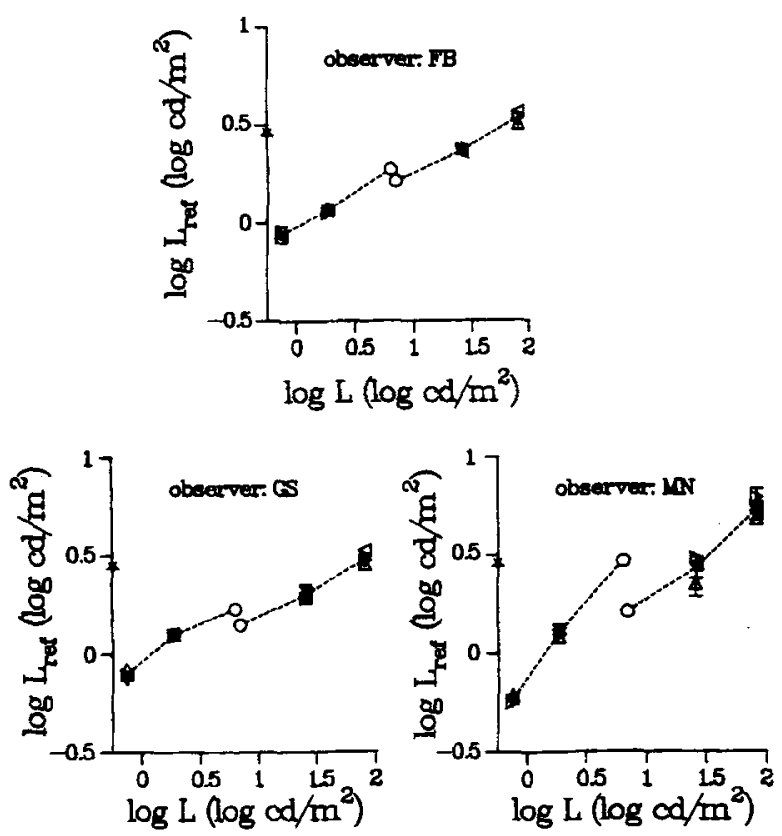

Figure A2. Results of the color control experiment for $\mathrm{Ob}-$ servers F.B., G.S., and M.N. The luminance of the scene elements of the test stimuli are plotted along the horizontal axis. The vertical axis represents the luminance of the reference disk; the background luminance of this disk is marked by a star. The triangles, used for the disks in the test stimuli, represent geometrical means of four matches. A right-pointing triangle refers to Color Point $A$, an upward-pointing triangle to Color Point $B$, a left-pointing triangle to Color Point $C$, and a downward-pointing triangle to Color Point $D$. The circles refer to brightness matches of the Ganzfeld. Dashed lines connect averaged $\log L_{\text {ref }}$ values of equiluminant scene elements.

larly in the case of test disks that were brighter than the Ganzfeld, such color differences were experienced as annoying. Second, it should be mentioned that small color shifts of the test stimuli can be introduced easily via the filters $F_{1}$ and $F_{2}$ (see Apparatus section). Because decreasing $\kappa$ in Equation 1 meant "put identical filters in cascade," changes of the attenuation were, in practice, accompanied by gradual color shifts.

With careful calibration, an inventory was made of the color coordinates occurring in the gamut of scene elements and $\kappa$ values of the patterns $P_{1}, P_{2}$, and $P_{3}$. The ellipse in the CIE chromaticity diagram of Figure Al only just encloses all these color points. For the control experiment, four points on the boundary of this confined region, labeled A, B, C, and D, were selected. The color point of the reference disk coincides with Point $A$ in this diagram. Two patterns, $P_{1}$ and $P_{3}$ (see Figure 5), with approximately equal Ganzfeld luminances, were taken. This gave a set of eight test stimuli, viz. $\left\{\mathrm{P}_{1, \mathrm{~A}}, \mathbf{P}_{1, \mathrm{~B}}, \mathrm{P}_{1, \mathrm{C}}, \mathbf{P}_{1, \mathrm{D}}, \mathrm{P}_{3, \mathrm{~A}}, \mathbf{P}_{3, \mathrm{~B}}\right.$, $\left.P_{3, C}, P_{3, D}\right\}$. For example, in conformity with pattern $P_{1}$, the test stimulus $P_{1, C}$ consisted of a Ganzfeld on which two bright disks with Color Point $C$ were superimposed. The match procedure described in the General Procedure section was followed. During a session, which lasted about $45 \mathrm{~min}$, one brightness match was made for every scene element of the eight test stimuli. The sequence of scene elements and test stimuli was randomized over the sessions. Four sessions were run for every observer.

The results of this color control experiment for Observers F.B., G.S., and M.N. are shown in Figure A2. As in Figure 6, lu- 
minances of the various scene elements of the test stimuli are plotted along the abscissa. Again, the ordinate represents the luminance of the reference disk. The background luminance of the reference stimulus is denoted by a star. The triangles, used for the disks in the test stimuli, represent geometrical means of four matches; standard deviations of these means are indicated by vertical bars. Because the brightness matches of the Ganzfelder, on the one hand of the subset $\left\{\mathrm{P}_{1, \mathrm{~A}}, \mathrm{P}_{1, \mathrm{~B}}, \mathrm{P}_{1, \mathrm{C}}, \mathrm{P}_{1, \mathrm{D}}\right\}$, and on the other hand of the subset $\left\{P_{3, A}, P_{3, B}, P_{3, C}, P_{3, D}\right\}$, are very much alike, only the geometrically averaged value of each subset, designated by a circle, is plotted. Like an arrow on the hand of a watch, right-pointing triangles are used for disks with Color Point A, upward-pointing triangles for disks with Color Point B, leftpointing triangles for disks with Color Point $C$, and downwardpointing triangles for disks with Color Point $D$.

The graphs in Figure A2 reveal, at least for these four color points, that there is no significant effect of color on brightness. In this respect, the color-weak observers F.B. and G.S. do not behave differently from Observer M.N., who has normal color vision. Note, moreover, that the Ganzfeld brightness of Pattern $P_{1}$ is slightly lower than the Ganzfeld brightness of Pattern $P_{3}$.

\section{APPENDIX B}

\section{Control Experiment 2: A General Check}

A general control possibility for the experimental procedure and the apparatus can be accomplished by stimulating both eyes of the viewer with exactly the same visual information. It is obvious that two equivalent stimuli, presented sequentially to the left and the right eye, must evoke similar brightness impressions if there are no differences in sensitivity between the stimulated pathways. For this second control experiment, the equivalence was achieved in two steps. First, the spatial configuration of the test stimuli was made identical to that of the reference stimulus (see Figure 3), while the time sequence remained as depicted in Figure 4 . This is abbreviated to SC (spatial correspondence). Subsequently, the temporal structure of presentation was altered. The time sequence of the reference stimulus was applied to the test stimuli with a delay of $2 \mathrm{sec}$. Thus at each trial, the test stimuli were also visible for only $1 \mathrm{sec}$. This alteration is henceforth referred to as STC (spatiotemporal correspondence).

The dark surround of the test stimuli was created by means of an opaque cylinder-cone shape that fitted into the integrating sphere. The luminance of the resulting $9^{\circ}$ background field, $L_{0, \text { test }}$, was set close to the fixed level $L_{0, \text { ref. }}$ For the central $1.2^{\circ}$ disk of the test stimuli, five luminance values $L_{\text {test }}$ around the constant background luminance $L_{0 \text {,test were taken. Apart from }}$ the time sequence employed for the STC condition, the procedure described in the General Procedure section was adhered to. In each session, which took about $30 \mathrm{~min}$, four brightness matches were obtained for every $L_{\text {test }}$ value, one concerned with the test disk for the SC condition, one with the background field for the SC condition, one with the test disk for the STC condition, and one with the background field for the STC condition. Again, four sessions were run for Observers F.B., G.S., and M.N. In two sessions, the luminance $L_{\text {test }}$ of the test disk was gradually increased; in the other two sessions, this order was reversed.

In Figure B1, the results for Observers F.B., G.S., and M.N. are plotted against the luminance $L_{\text {test }}$ of the test disk. Since an increasing or decreasing order of the luminance $L_{\text {test }}$ leads to similar results, only $\log L_{\text {ref }}$ values averaged over four sessions are presented in the plot. Standard deviations of these means are smaller than the symbol size. For the STC condition, upwardpointing triangles and diamonds halved by a horizontal line, both connected by a dashed curve, denote the brightness matches of
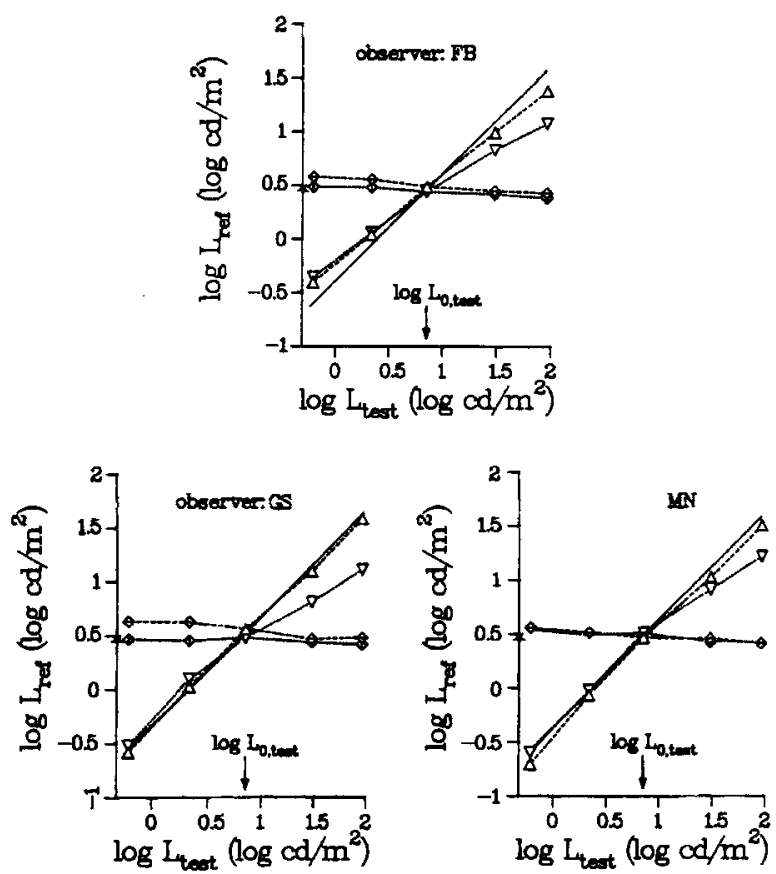

Figure B1. Results of the general control experiment for $\mathrm{Ob}$ servers F.B., G.S., and M.N. The luminance $L_{\text {ref }}$ of the reference disk is plotted against the luminance $L_{\text {test }}$ of the test disk in $\log$-log units. The background luminance of the reference stimulus is denoted by a star on the ordinate; the background luminance $L_{0, \text { test }}$ of the test stimuli is indicated by an arrow in the plot. For the STC condition, upward-pointing triangles and diamonds halved by a horizontal line, connected with dashed curves, refer, respectively, to the test disk and its background field. For the SC condition, downward-pointing triangles and diamonds halved by a vertical line, connected with dotted curves, refer, respectively, to the test disk and its background field.

the test disk and its background field, respectively. For the SC condition, downward-pointing triangles and diamonds halved by a vertical line, connected by dotted curves, are used for the test disk and its background field, respectively.

In the case of STC, the brightness matches related to the test disk do not differ significantly from the solid line with slope 1.0, at least for Observers G.S. and M.N. In other words, equal relative luminance increments (or decrements) of test and reference disk, presented for the same period of time to the left and right eye, respectively, result in similar brightness values attributed to these disks. Therefore, we can conclude from this general control experiment that the results comply with commonsense expectations. This validates the experimental procedure employed and indicates that the cumbrous structure of the optical apparatus sketched in Figure 2 gives no reason to suspect that any effects have been overlooked. Furthermore, brightness matches for test stimuli, with disk luminances $L_{\text {test }}<L_{0 \text {, test }}$, are nearly identical for SC and STC conditions. On the other hand, if $L_{\text {lest }}>$ $L_{0, \text { test }}$, differences arise between the SC and STC conditions, indicating that temporal aspects play a role in brightness perception. A way to handle this is to use weights $\alpha$ in the model that depend on the temporal structure, the contrast polarity, and, of course, the illumination level.

(Manuscript received March 24, 1994; revision accepted for publication February 24, 1995.) 